\title{
Coupling impedance of many holes in a liner within a beam pipe
}

\author{
Robert L. Gluckstern \\ Department of Physics, University of Maryland, College Park, Maryland 20742
}

(Received 26 February 1992)

\begin{abstract}
The longitudinal and transverse impedance of many holes in a thick-wall liner have been calculated for hole dimensions that are small compared to the wavelength. These are shown to be small compared with the corresponding resistive wall impedances of the liner at the frequency of the lowest transverse coupled-bunch mode. Estimates are made of the effect of the propagation of TEM modes in the coaxial region between the liner and beam pipe, and these can be neglected for a sufficiently thick liner. Estimates are also made of the possible coherent effect of uniformly spaced holes.

PACS number(s): 41.20.-q, 03.40.Kf, 03.50.De, 41.75.-i
\end{abstract}

\section{INTRODUCTION}

In the preceding paper [1] the coupling impedance of a single hole in a beam pipe was calculated for hole dimensions small compared to the wavelength. In the present paper we analyze the situation where a liner with many holes is placed inside a beam pipe. This configuration is being considered to remove the heat generated by the synchrotron radiation of a high-intensity beam in a highenergy proton storage ring, while allowing a low vacuum to be maintained within the liner.

We first consider the case where the fields in the coaxial region between the liner of radius $b$ and the beam pipe of radius $a$ can be neglected, and where none of the possible modes within the liner travel with the velocity of light (in synchronism with the drive beam). We then remove these assumptions and show that the results are not appreciably changed for a reasonable choice of parameters, even if the spacing between the holes is uniform.

\section{ANALYSIS FOR COUPLING IMPEDANCE}

The longitudinal coupling impedance is most easily obtained from the expansion of $E_{z}$, the axial electric field within the liner. Specifically we write

$E_{z}(r, \theta, z ; k)=\sum_{n} \int_{-\infty}^{\infty} d q e^{-j q z-j n \theta} A_{n}(q) \frac{J_{n}(K r)}{J_{n}(K b)}$

where

$$
K^{2}=k^{2}-q^{2}
$$

and where the contour goes below any poles on the negative real $q$ axis and above any poles on the positive real $q$ axis. The longitudinal coupling impedance can be written in terms of $A_{n}(q)$ as [1]

$$
Z_{\|}(k)=-\frac{2 \pi}{Q} A_{0}(k)
$$

where $Q$ is the source charge. The inverse transform of Eq. (2.1) leads to

$$
Z_{\|}(k)=-\frac{1}{2 \pi b Q} \iint d S e^{j k z} E_{z}(b, \theta, z ; k),
$$

where the surface integral is now taken over the interior openings of all holes in the beam pipe. Assuming that each hole is small, we may take the unperturbed source fields for $E_{r}$ and $H_{\theta}$. Furthermore, if the wavelength is large compared to the hole size, we can use a quasistatic analysis for the induced electric and magnetic dipole moments [2]. We then obtain the same results as for a single hole, but for the present situation we must sum over all $P$ holes. Thus

$$
\frac{Z_{\|}(k)}{Z_{0}}=\frac{j k}{8 \pi^{2} b^{2}}\left(\psi_{\mathrm{in}}-\chi_{\mathrm{in}}\right) P
$$

where $\psi_{\text {in }}$ and $\chi_{\text {in }}$ are the inside susceptibility (in the $\theta$ direction) and polarizability of the holes [3]. For harmonic $n=k R$, where $R$ is the radius of the ring, one finds

$$
\frac{Z_{\|}(k)}{n Z_{0}}=\frac{j \eta\left(\psi_{\mathrm{in}}-\chi_{\mathrm{in}}\right)}{2 b \Delta},
$$

where $\eta$ is the fraction of the liner surface covered by holes, and where $\Delta$ is the cross-sectional area of each hole.

For comparison, we use a similar calculation to obtain the well-known longitudinal coupling impedance for the resistive wall [4]. In this case the integral in Eq. (2.4) is evaluated using

$$
E_{z}=-Z_{0} H_{\theta} \frac{k \delta}{2}(1+j),
$$

where $\delta$ is the skin depth of the interior liner surface. Using the source magnetic field

$$
Z_{0} H_{\theta}=\frac{Q e^{-j k z}}{2 \pi b}
$$

we find the result for the longitudinal impedance of the resistance wall

$$
\frac{Z_{\|}^{\mathrm{RW}}(k)}{n Z_{0}}=\frac{1+j}{2} \frac{\delta}{b}
$$


As a practical matter, we want the contributions from the holes to be smaller than that from the resistive wall. Thus we need to restrict the liner surface fraction $\eta$ to a value determined by the condition

$$
\frac{\eta\left(\psi_{\text {in }}-\chi_{\text {in }}\right)}{\Delta \delta}<1
$$

For circular holes of radius $r_{0}$, this reduces to [3]

$$
\frac{\eta r_{0}}{4 \delta}<1
$$

A similar analysis for the transverse coupling impedance [1] leads to

$$
\frac{Z_{\perp}(k)}{Z_{0}}=\frac{j \eta\left(\psi_{\mathrm{in}}^{(0)}-\chi_{\mathrm{in}}\right)}{2 b \Delta} \frac{2 R}{b^{2}}
$$

Since the transverse resistive wall impedance also satisfies

$$
Z_{\perp}=\frac{Z_{\|}}{n} \frac{2 R}{b^{2}}
$$

the same considerations as before also apply to the relative contributions of the transverse impedance caused by the holes and by the wall resistance.

The most serious resistive wall effect corresponds to the lowest frequency for the transverse coupled-bunch modes. For this purpose the CERN Large Hadron Collider (LHC) design assumes $\delta=0.1 \mathrm{~mm}$. With a hole radius $r_{0}=1$ $\mathrm{mm}$, Eq. (2.11) implies that $\eta<0.4$. In the current design with $\eta=0.05$, which is apparently sufficient to maintain a low vacuum within the liner, the contribution of the holes to either the longitudinal or the transverse impedance will be about $1 / 8$ of the corresponding resistive wall coupling impedance at the frequency corresponding to the lowest coupled-bunch mode.

\section{TEM MODES IN THE COAXIAL REGION}

In the previous section we ignored the fields in the coaxial region between the outside surface of the liner and the inside surface of the beam pipe of radius $a$. These fields have the potential to be important, since in this region the TEM mode in the forward direction travels at the velocity of light, in synchronism with the source charge.

The longitudinal component of the electric field in the coaxial region can be written as

$E_{z}(r, \theta, z ; k)=\sum_{n} \int_{-\infty}^{\infty} d q e^{-j q z-j n \theta} B_{n}(q) \frac{F_{n}(K r)}{F_{n}(K b)}$

where the linear combination of Bessel functions

$$
F_{n}(u)=Y_{n}(u) J_{n}(K a)-J_{n}(u) Y_{n}(K a)
$$

is chosen to satisfy the boundary condition at $r=a$. (We consider the inside and outside surfaces of the liner to both be at $r=b$, since we neglect the thickness of the liner compared to the wavelength.) Inverting Eqs. (2.1) and (3.1) leads to

$$
\begin{aligned}
& A_{n}(q)=\frac{1}{4 \pi^{2} b} \iint_{\text {inside }} d S E_{z}\left(b_{-}, \theta, z ; k\right) e^{j q z+j n \theta} \\
& B_{n}(q)=\frac{1}{4 \pi^{2} b} \iint_{\text {outside }} d S E_{z}\left(b_{+}, \theta, z ; k\right) e^{j q z+j n \theta} .
\end{aligned}
$$

If the liner were of zero thickness $B_{n}(q)$ and $A_{n}(q)$ would be the same since $E_{z}$ would be continuous at $r=b$ within the holes and on both sides of the liner surface, where $E_{z}=0$. For a thick liner this is not the case.

We again treat the problem as quasistatic in the hole region where $E_{r}, H_{\theta}$ are denoted by $E_{1}, H_{1}$ near the inside surface of the hole and by $E_{2}, H_{2}$ near the outside surface. For the electric field the situation is as shown in Fig. 1. In Fig. 2 we separate the calculation into one which is symmetric and one which is antisymmetric in the electrical potential. The configuration in Fig. 1 is then the sum of those in Fig. 2. The solution to the electrostatic and magnetostatic problems allows us then to write, for hole $m$

$$
\begin{aligned}
& A_{n}(q)=\frac{j}{8 \pi^{2} b}\left(C_{s}+C_{a}\right) e^{j q z_{m}+j n \theta_{m}}, \\
& B_{n}(q)=\frac{j}{8 \pi^{2} b}\left(C_{s}-C_{a}\right) e^{j q z_{m}+j n \theta_{m}}
\end{aligned}
$$

where

$$
\begin{aligned}
& C_{s}=q \chi_{s}\left(E_{1}-E_{2}\right)-k Z_{0} \psi_{s}\left(H_{1}-H_{2}\right)_{\theta}, \\
& C_{a}=q \chi_{a}\left(E_{1}+E_{2}\right)-k Z_{0} \psi_{a}\left(H_{1}+H_{2}\right)_{\theta} .
\end{aligned}
$$

Here $z_{m}, \theta_{m}$ denote the location of the center of the hole, and $\chi_{s, a}, \psi_{s, a}$ are the symmetric, antisymmetric polarizability and susceptibility. In terms of the inside and outside polarizability and susceptibility defined by

$$
\chi_{\text {in }, \text { out }} \equiv \chi_{s} \pm \chi_{a} \quad, \quad \psi_{\text {in,out }} \equiv \psi_{s} \pm \psi_{a},
$$

we have

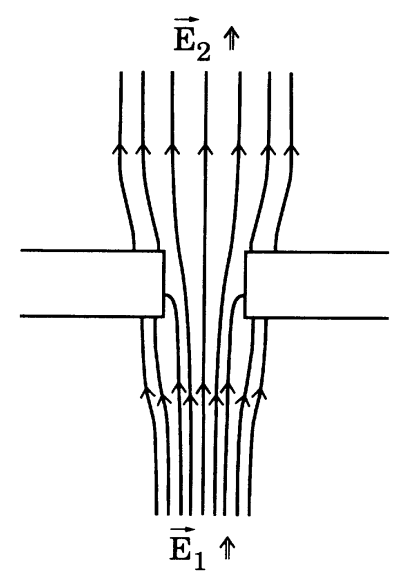

FIG. 1. Static electric-field configuration near a hole in a thick-wall beam pipe. 

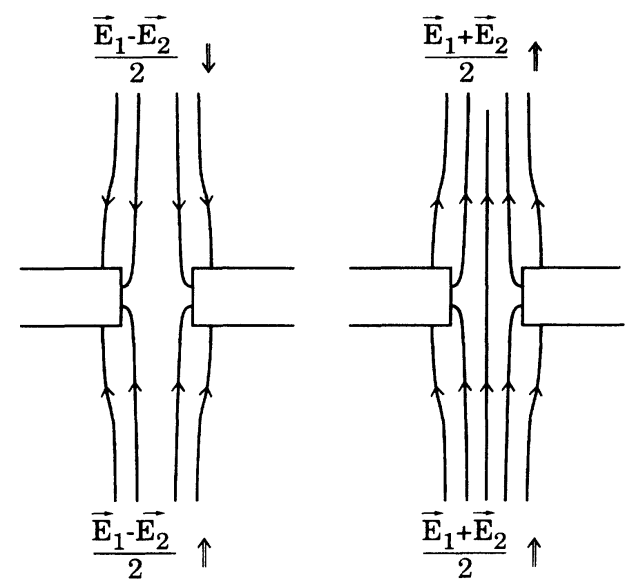

FIG. 2. Separation into symmetric and antisymmetric electrostatic potential configurations.

$$
\begin{aligned}
& A_{n}(q)=\frac{j}{8 \pi^{2} b} \sum_{m}\left[q E_{1} \chi_{\text {in }}-q E_{2} \chi_{\text {out }}\right. \\
& \left.-k Z_{0} H_{1} \psi_{\text {in }}+k Z_{0} H_{2} \psi_{\text {out }}\right]_{m} \\
& \times e^{j q z_{m}+j n \theta_{m}}, \\
& B_{n}(q)=\frac{j}{8 \pi^{2} b} \sum_{m}\left[q E_{1} \chi_{\text {out }}-q E_{2} \chi_{\text {in }}\right. \\
& \left.-k Z_{0} H_{1} \psi_{\text {out }}+k Z_{0} H_{2} \psi_{\text {in }}\right]_{m} \\
& \times e^{j q z_{m}+j n \dot{\theta}_{m}}
\end{aligned}
$$

where we now sum over all holes.

We now write the field components $E_{r}, H_{\theta}$ in the coaxial region as

$$
\begin{gathered}
\left\{\begin{array}{c}
E_{r} \\
Z_{0} H_{\theta}
\end{array}\right\}=-j \sum_{n} \int_{-\infty}^{\infty} d q\left\{\begin{array}{l}
q \\
k
\end{array}\right\} \times e^{-j q z-n \theta} \\
\times B_{n}(q) \frac{F_{n}^{\prime}(K r)}{K F_{n}(K b)} \\
+(\text { TE modes }),
\end{gathered}
$$

where we have written only the TM modes explicitly. There are poles at $q= \pm k\left(K^{2}=0\right)$ only for the $n=0$ term in the TM modes, corresponding to the TEM modes traveling with the velocity of light in both the positive and negative $z$ directions. If we assume that the holes are spaced so that only the TEM mode in the forward direction can build up, we find, near the pole at $q=k$,

$$
\frac{F_{0}^{\prime}(K r)}{K F_{0}(K b)} \cong \frac{1}{q^{2}-k^{2}} \frac{1}{r \ln (a / b)}
$$

The contribution from this pole comes only when the contour is closed in the lower-half $q$ plane. It is nonvanishing only when $z_{m}<z$ and leads in the coaxial region to

$$
\begin{aligned}
E_{r}=Z_{0} H_{\theta} & \\
=-\frac{j k}{8 \pi b r \ln (a / b)} \sum_{z_{m}<z} & e^{-j k\left(z-z_{m}\right)} \\
& \begin{array}{l}
\times\left[E_{1} \chi_{\text {out }}-E_{2} \chi_{\text {in }}-Z_{0} H_{1} \psi_{\text {out }}\right. \\
+
\end{array} \\
& \left.+Z_{0} H_{2} \psi_{\text {in }}\right]_{m} .
\end{aligned}
$$

We recognize this as the TEM mode in the coaxial region, and see that the rate of buildup of this mode depends on the extent to which it has already built up. In fact, since

$$
\left(E_{1}\right)_{m}=Z_{0}\left(H_{1}\right)_{m}=\frac{Q e^{-j k z_{m}}}{2 \pi b}
$$

we can set $E_{r}$ and $H_{\theta}$ equal to $E_{2}$ and $H_{2}$ at $r=b$ and write

$$
E_{r}=Z_{0} H_{r}=\left(E_{2}\right)_{m}=Z_{0}\left(H_{2}\right)_{m} \equiv \frac{Q e^{-j k z_{m}}}{2 \pi b} F_{m} .
$$

In this way we obtain, for identical holes,

$$
\begin{aligned}
F_{m^{\prime}}= & \frac{j k}{8 \pi b^{2} \ln (a / b)} \\
& \times \sum_{m<m^{\prime}}\left[\left(\psi_{\text {out }}-\chi_{\text {out }}\right)\right. \\
& \left.\quad-F_{m}\left(\psi_{\text {in }}-\chi_{\text {in }}\right)\right] e^{-\alpha\left(z_{m^{\prime}}-z_{m}\right)},
\end{aligned}
$$

where we have included the attenuation constant $\alpha$ of the TEM mode in the coaxial region. We now calculate the steady-state level reached by $F_{m}$ when the average axial hole spacing is $L$. In this way we find

$$
F=\frac{j k\left(\psi_{\text {out }}-\chi_{\text {out }}\right)}{8 \pi b^{2} \alpha L \ln (a / b)+j k\left(\psi_{\text {in }}-\chi_{\text {in }}\right)} .
$$

It is easy to see that the longitudinal impedance in Eq. (2.5) must now be modified to include the effect of the steady-state field in the coaxial region. From the form for $A_{0}(k)$ in Eq. (3.10) we see that this leads to the replacement

$$
\psi_{\text {in }}-\chi_{\text {in }} \rightarrow \psi_{\text {in }}-\chi_{\text {in }}-F\left(\psi_{\text {out }}-\chi_{\text {out }}\right)
$$

in Eq. (2.6) and we have

$$
\begin{aligned}
\frac{Z_{\|}(k)}{n Z_{0}}=\frac{j \eta}{2 \Delta b}[ & \psi_{\text {in }}-\chi_{\text {in }} \\
& \left.-\frac{\left(\psi_{\text {out }}-\chi_{\text {out }}\right)^{2}}{\psi_{\text {in }}-\chi_{\text {in }}-j 8 \pi b^{2}(\alpha / k) L \ln (a / b)}\right]
\end{aligned}
$$

We complete this phase of the calculation by expressing the attenuation constant in terms of the geometry and wall resistivity in the coaxial region. Specifically

$$
\frac{\alpha \ln (a / b)}{k}=\frac{1}{4}\left(\frac{\delta_{a}}{a}+\frac{\delta_{b}}{b}\right),
$$


where we allow for the possibility of different wall resistivities in the coaxial region.

The frequencies at which the skin depths should be calculated are in the $\mathrm{GHz}$ range, corresponding to the longitudinal bunch shape. At these frequencies and with $\eta=0.05$, the term involving the attenuation in Eq. (3.18) can be neglected and we find

$$
F \cong \frac{\psi_{\text {out }}-\chi_{\text {out }}}{\psi_{\text {in }}-\chi_{\text {in }}}
$$

and

$$
\frac{Z_{\|}(k)}{n Z_{0}}=\frac{j \eta\left(\psi_{\text {in }}-\chi_{\text {in }}\right)}{2 b \Delta}\left[1-F^{2}\right]
$$

Thus the presence of the outer wall reduces the impedance. Values of the outside polarizability and susceptibility for a circular hole in a thick wall decrease rapidly with the wall thickness [3]. (For wall thickness greater than one hole radius, the reduction amounts to a factor 10 or greater.)

We make a few additional observations at this point.

(1) The relative level to which the fields build up in the coaxial region is given in Eq. (3.18). It is extremely small because of the exponential decrease of the numerator with increasing wall thickness.

(2) The result for the transverse coupling impedance is even simpler, since no transverse TEM mode with $\cos \theta$ dependence exists in the coaxial region. Thus the result in Eqs. (2.12) and (2.13) is not modified by the existence of the coaxial region.

(3) In the absence of losses, the impedance depends on the difference between $\left(\psi_{\text {in }}-\chi_{\text {in }}\right)^{2}$ and $\left(\psi_{\text {out }}-\chi_{\text {out }}\right)^{2}$. For zero wall thickness this difference vanishes and the steady-state impedance vanishes. In this case the field level in the coaxial region builds up to that within the liner, leading to no steady-state contribution to the impedance.

\section{UNIFORM HOLE SPACING}

In the previous sections we have assumed that the holes are spaced such that only modes traveling wth $v=c$ in the forward direction can remain in synchronism with the driving source and build up. In this section we consider the more attractive practical situation of uniform hole spacing and ask if the coherent buildup of other modes will modify our results.

We start with the equivalent of Eq. (3.12) in the region within the liner and consider, as an example, a propagating azimuthally independent $\mathrm{TM}_{0 \ell}$ mode, for which we write

$$
\begin{aligned}
\left\{\begin{array}{c}
E_{r} \\
Z_{0} H_{\theta}
\end{array}\right\}= & -j \int_{-\infty}^{\infty} d q\left\{\begin{array}{l}
q \\
k
\end{array}\right\} \times e^{-j q z} A_{0}(q) \frac{J_{0}^{\prime}(K r)}{K J_{0}(K b)} \\
& +\frac{Q}{2 \pi r} e^{-j k z}
\end{aligned}
$$

where we include the driving source term. Neglecting the fields in the coaxial region, we write, as in Eq. (3.10),

$$
A_{0}(q)=\frac{j}{8 \pi^{2} b} \sum_{m}\left(q E_{m} \chi_{\mathrm{in}}-k Z_{0} H_{m} \psi_{\mathrm{in}}\right) e^{j q z_{m}}
$$

where $E_{m}, H_{m}$ are the radial electric and azimuthal magnetic fields within the liner near the $m$ th hole.

Let us now consider the pole at $K b=p_{\ell}$ in Eq. (4.1), where $J_{0}\left(p_{\ell}\right)=0$. Near $r=b$ and $K b=p_{\ell}$ we write

$\frac{J_{0}^{\prime}(K r)}{K J_{0}(K b)} \cong \frac{1}{K\left(K b-p_{\ell}\right)} \cong-\frac{2}{b\left(q^{2}-c_{\ell}^{2} / b^{2}\right)}$

where

$$
c_{\ell}=\left(k^{2} b^{2}-p_{\ell}^{2}\right)^{1 / 2} .
$$

Combining Eqs. (4.1)-(4.3) and evaluating the residues at the poles in the $q$ plane, we obtain

$$
\left.\begin{array}{c}
E_{r} \\
Z_{0} H_{\theta}
\end{array}\right\}=\frac{Q e^{-j k z}}{2 \pi b}+\frac{j}{4 \pi b^{3} c_{\ell}} \times\left\{\begin{array}{c}
c_{\ell} \\
k b
\end{array}\right\} \times \sum_{z_{m}<z}\left(c_{\ell} E_{m} \chi_{\mathrm{in}}-k b Z_{0} H_{m} \psi_{\mathrm{in}}\right) e^{-j\left(c_{\ell} / b-j \alpha\right)\left(z-z_{m}\right)}
$$

where we retain only the forward traveling mode $\ell$. A small, positive imaginary part has been added to $c_{\ell}$ in the exponent, corresponding to the attenuation of this mode. We now write

$$
\left.\begin{array}{c}
E_{r} \\
Z_{0} H_{\theta}
\end{array}\right\}=\frac{Q e^{-j k z}}{2 \pi b} \times\left\{\begin{array}{c}
F \\
G
\end{array}\right\}
$$

and obtain, for uniform hole spacing, the difference equations

$$
\begin{aligned}
& \left.\begin{array}{l}
F_{m^{\prime}} \\
G_{m^{\prime}}
\end{array}\right\}=1-\frac{n_{\theta}}{4 \pi b^{3} c_{\ell}} \times\left\{\begin{array}{l}
c_{\ell} \\
k b
\end{array}\right\} \\
& \times \sum_{\substack{m \\
m<m^{\prime}}}\left(k b G_{m} \psi_{\mathrm{in}}-c_{\ell} F_{m} \chi_{\mathrm{in}}\right) \\
& \times e^{\left(j \theta-\alpha L_{z}\right)\left(m^{\prime}-m\right)},
\end{aligned}
$$

where

$$
\theta=\left(k b-c_{\ell}\right) L_{z} / b \quad(\text { modulus } 2 \pi),
$$


and where $n_{\theta}$ is the number of holes equally spaced in the azimuthal direction at a given axial location. Here $L_{z}$ is the axial spacing between rings of holes.

We now solve for the asymptotic behavior of $F$ and $G$, which differs from 1 only when $\left|\alpha L_{z}-j \theta\right| \ll 1$. (We subtract the nearest multiple of $2 \pi$ from $\theta$.) In this way we find, for small $\theta$ and $\alpha L_{z}$,

$$
\left.\begin{array}{l}
F \\
G
\end{array}\right\}=1-\frac{j n_{\theta}\left(k b G \psi_{\mathrm{in}}-c_{\ell} F \chi_{\mathrm{in}}\right)}{4 \pi b^{3} c_{\ell}\left(\alpha L_{z}-j \theta\right)} \times\left\{\begin{array}{l}
c_{\ell} \\
k b
\end{array},\right.
$$

from which we see that

$$
\begin{aligned}
& \left(k b G \psi_{\mathrm{in}}-c_{\ell} F \chi_{\mathrm{in}}\right) \\
& \quad=\frac{n_{\theta}\left(k b \psi_{\mathrm{in}}-c_{\ell} \chi_{\mathrm{in}}\right) 4 \pi b^{3} c_{\ell}\left(\alpha L_{z}-j \theta\right)}{4 \pi b^{3} c_{\ell}\left(\alpha L_{z}-j \theta\right)+j n_{\theta}\left(k^{2} b^{2} \psi_{\mathrm{in}}-c_{\ell}^{2} \chi_{\mathrm{in}}\right)} .
\end{aligned}
$$

The impedance is obtained in terms of the asymptotic fields $E_{r}$ and $H_{\theta}$ as it was in Eq. (2.5), and is

$$
\frac{Z_{\|}(k)}{Z_{0}}=\frac{j k P}{8 \pi^{2} b^{2}}\left(\psi_{\mathrm{in}} G-\chi_{\mathrm{in}} F\right) .
$$

Using Eqs. (4.9)-(4.11) we find

$$
\begin{aligned}
\frac{Z_{\|}(k)}{Z_{0}}=\frac{j k P}{8 \pi^{2} b^{2}}[ & \left(\psi_{\mathrm{in}}-\chi_{\mathrm{in}}\right) \\
& \left.\quad-\frac{j n_{\theta}\left(k b \psi_{\mathrm{in}}-c_{\ell} \chi_{\mathrm{in}}\right)^{2}}{4 \pi b^{3} c_{\ell}\left(\alpha L_{z}-j \theta+j \theta_{0}\right)}\right],
\end{aligned}
$$

where

$$
\theta_{0} \equiv \frac{n_{\theta}\left(k^{2} b^{2} \psi_{\mathrm{in}}-c_{\ell}^{2} \chi_{\mathrm{in}}\right)}{4 \pi b^{3} c_{\ell}}
$$

Thus there is a resonantlike effect due to the uniform hole spacing which reaches its maximum value when $\theta=\theta_{0}$ and which has a half-width

$$
\Delta \theta=\alpha L_{z} .
$$

At synchronism we have

$$
\frac{Z_{\|}(k)}{n Z_{0}}=\frac{\eta}{2 \Delta b}\left[j\left(\psi_{\mathrm{in}}-\chi_{\mathrm{in}}\right)+\frac{n_{\theta}\left(k b \psi_{\mathrm{in}}-c_{\ell} \chi_{\mathrm{in}}\right)^{2}}{4 \pi b^{3} c_{\ell} \alpha L_{z}}\right],
$$

where the real part of the impedance is now associated with the wall dissipation.

A similar analysis for a backward $\mathrm{TM}_{0 \ell}$ mode within the liner leads to almost the same result. In this case the phase shift between adjacent axial locations is

$$
\theta=\left(k b+c_{\ell}\right) L_{z} / b \quad(\text { modulus } 2 \pi)
$$

and the longitudinal impedance becomes

$$
\frac{Z_{\|}(k)}{Z_{0}}=\frac{j k P}{8 \pi b^{2}}\left[\left(\psi_{\mathrm{in}}-\chi_{\mathrm{in}}\right)-\frac{j n_{\theta}\left(k b \psi_{\mathrm{in}}+c_{\ell} \chi_{\mathrm{in}}\right)^{2}}{4 \pi b^{3} c_{\ell}\left(\alpha L_{z}-j \theta-j \theta_{0}\right)}\right],
$$

corresponding to a resonance at $\theta=-\theta_{0}$ with a halfwidth given again by Eq. (4.14).

The frequencies at which this coherence occurs correspond to $\theta=2 m \pi$ with $m=1,2, \ldots$. From Eqs. (4.8) and (4.16) these correspond to

$$
k=\frac{m \pi}{L_{z}}\left[1+\left(\frac{p_{\ell} L_{z}}{2 m \pi b}\right)^{2}\right], m=1,2, \ldots
$$

In addition, the relative width of such a resonance is

$$
\frac{\Delta k}{k}=\frac{\Delta \theta}{\theta}\left[1-\frac{p_{\ell}^{2}}{k^{2} b^{2}}\right]^{1 / 2}=\frac{\alpha L_{z} c_{\ell}}{2 m \pi k b} .
$$

Since the attenuation constant of the $\mathrm{TM}_{0 \ell}$ mode is

$$
\alpha=k^{2} \delta / 2 c_{\ell},
$$

the effective $Q_{r}$ of this resonance can be written as

$$
Q_{r}=\frac{4 b}{\delta}\left[1+\left(\frac{L_{z} p_{\ell}}{2 m \pi b}\right)^{2}\right]^{-1}
$$

For a skin depth $\delta$ corresponding to the $\mathrm{GHz}$ frequency range, $Q_{r}$ is of order $10^{5}$.

At synchronism we have, from Eqs. (4.15) and (4.17),

$$
\frac{Z_{\|}(k)}{n Z_{0}}=\frac{\eta}{2 b \Delta}\left[j\left(\psi_{\mathrm{in}}-\chi_{\mathrm{in}}\right)+\frac{n_{\theta}\left(k b \psi_{\mathrm{in}} \mp c_{\ell} \chi_{\mathrm{in}}\right)^{2}}{4 \pi b^{3} c_{\ell} \alpha L_{z}}\right],
$$

where the real part of the impedance is now associated with the wall dissipation. Using $L_{z}=n_{\theta} \Delta / 2 \pi b \eta$ and the expressions for $\alpha$ in Eq. (4.20) and $Q_{r}$ in (4.21), we can write for the impedance at synchronism

$$
\begin{aligned}
\frac{Z_{\|}(k)}{n Z_{0}}= & \frac{j \eta\left(\psi_{\mathrm{in}}-\chi_{\mathrm{in}}\right)}{2 b \Delta} \\
& +\left[\frac{\eta\left(\psi_{\mathrm{in}} \mp \chi_{\mathrm{in}} c_{\ell} / k b\right)}{2 b \Delta}\right]^{2} \frac{Q_{r}}{2} \\
& \times\left[1+\left(\frac{p_{\ell} L_{z}}{2 m \pi}\right)^{2}\right] .
\end{aligned}
$$

If we use the asymptotic value for a circular hole in a thick wall [1] given by

$$
\psi_{\text {in }}-\chi_{\text {in }}=0.562\left(4 r_{0}^{3} / 3\right),
$$

we find

$$
\frac{\eta\left(\psi_{\mathrm{in}}-\chi_{\mathrm{in}}\right)}{2 b \Delta} \simeq 0.12 \frac{\eta r_{0}}{b}
$$

which is approximately $3 \times 10^{-4}$ for the LHC parameters $\left(\eta=0.05, r_{0} / b=0.05\right)$. Since $L_{z}$ is of the order of a few $\mathrm{mm}$, we can neglect $p_{\ell}$ with respect to $k b$ and $b / L_{z}$ in Eq. (4.22) for most of the modes $\ell$ up to cutoff. (In fact, near cutoff the order of magnitude of the second term is not changed by neglecting $p_{\ell}$.) As a result, the second term in Eq. (4.22) is of order

$$
\left(3 \times 10^{-4}\right)^{2} \times 10^{5} \sim 10^{-2} .
$$


Thus the second term contributes a peak impedance of the order of $Z_{\|}(k) / n \simeq 4 \Omega$ with a width corresponding to a $Q_{r}$ of $10^{5}$.

The narrow width and high frequency of this mode, together with the bunch length of $5-10 \mathrm{~cm}$ reduces the effective impedance for the single bunch mode by a factor of 100 or so. But the situation is not really as serious as indicated here since the axial spacing between holes will not be constant to an accuracy of 1 part in $10^{5}$. Normal fabrication errors (or an intentional spread in the axial spacing) are likely to reduce $Q_{r}$ to a value more like $10^{2}$ and the corresponding contribution to the impedance will be of the order of $0.01 \Omega$ or less.

Thus we conclude that the second term in Eqs. (4.12) and (4.16) can be neglected and that coherence effects can be kept small. Similar conclusions apply for TE modes, as well as for modes other than TEM in the coaxial region, and also for coherent propagation of deflecting modes.

\section{SUMMARY}

We have obtained the steady-state longitudinal and transverse coupling impedance of a liner with holes within a beam pipe for hole dimensions small compared to the wavelength. For a liner with thickness comparable with the hole dimensions the primary results are obtained neglecting all modes within the liner and in the coaxial region between the liner and the beam pipe. These results are compared to the corresponding contributions from the wall resistance and a simple limit is derived for the fraction of the liner surface that can be covered by holes in order that the impedance of the holes be less than the resistive wall impedance.

We then explore the contributions from all modes which may build up within the liner and in the coaxial region. The most serious for the longitudinal coupling impedance is the TEM mode in the coaxial region which is in synchronism with the drive beam. We show that the correction introduced by this mode and all other modes, which may be coherent for uniform hole spacing, will be smaller than the primary hole impedance result, provided that the axial hole spacing is not uniform to an accuracy of better than 1 part in 100 . (To be on the safe side it may be wise to make sure that such a nonunformity in hole spacing is built into the fabrication process.) Thus, the primary results for the impedance in Eqs. (2.6) and (2.12) accurately represent the physical situation.

We also believe that any reflections which may occur in the coaxial region by the mechanical supports for the liner can be neglected since they serve to further attenuate any coherent effects in the coaxial region.

\section{ACKNOWLEDGMENTS}

The author would like to thank J. Bisognano, F. Caspers, A. Chao, G. Dôme, J. Gayrete, F. Ruggiero, and B. Zotter for helpful comments and suggestions. He is also grateful to SL Division at CERN for its hospitality during the course of part of this work.
[1] R.L. Gluckstern, preceding paper, Phys. Rev. A 46, 1108 (1992).

[2] See, for example, H.A. Bethe, Phys. Rev. 66, 163 (1944).

[3] See, for example, R.L. Gluckstern and J.A. Diamond, IEEE Trans. Microwave Theory Tech. 39, 274 (1991).
[4] A. Chao, in Physics of High Energy Particle Accelerators [Stanford Linear Accelerator Center (SLAC) Stanford University, California], AIP Conf. Proc. No. 105, edited by Melvin Month (AIP, New York, 1982), p. 353. 